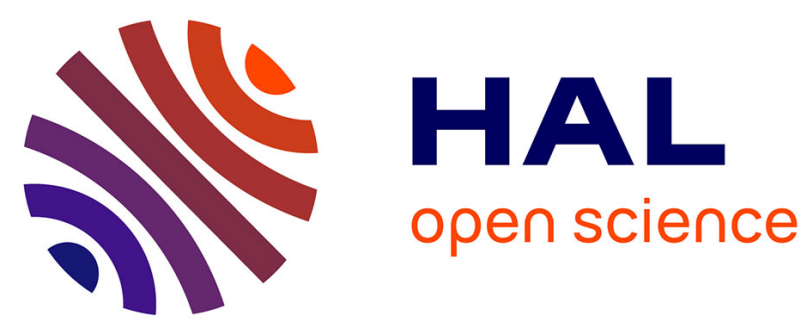

\title{
Intermittency in fractal Fourier hydrodynamics: Lessons from the Burgers equation
}

\author{
Uriel Frisch, Michele Buzzicotti, Luca Biferale, Samriddhi Sankar Ray
}

\section{To cite this version:}

Uriel Frisch, Michele Buzzicotti, Luca Biferale, Samriddhi Sankar Ray. Intermittency in fractal Fourier hydrodynamics: Lessons from the Burgers equation. Physical Review E , 2016, 93 (3), pp.033109. 10.1103/PhysRevE.93.033109 . hal-03527772

\section{HAL Id: hal-03527772 \\ https://hal.science/hal-03527772}

Submitted on 17 Jan 2022

HAL is a multi-disciplinary open access archive for the deposit and dissemination of scientific research documents, whether they are published or not. The documents may come from teaching and research institutions in France or abroad, or from public or private research centers.
L'archive ouverte pluridisciplinaire HAL, est destinée au dépôt et à la diffusion de documents scientifiques de niveau recherche, publiés ou non, émanant des établissements d'enseignement et de recherche français ou étrangers, des laboratoires publics ou privés. 


\title{
Intermittency in fractal Fourier hydrodynamics: Lessons from the Burgers equation
}

\author{
Michele Buzzicotti, ${ }^{1, *}$ Luca Biferale, ${ }^{1, \dagger}$ Uriel Frisch, ${ }^{2, \ddagger}$ and Samriddhi Sankar Ray ${ }^{3, \S}$ \\ ${ }^{1}$ Department of Physics and INFN, University of Rome “Tor Vergata," Via della Ricerca Scientifica 1, 00133, Rome, Italy \\ ${ }^{2}$ Lab. Lagrange, UCA, OCA, CNRS, CS, 34229, 06304, Nice Cedex 4, France \\ ${ }^{3}$ International Centre for Theoretical Sciences, Tata Institute of Fundamental Research, Bangalore 560089, India \\ (Received 16 October 2015; revised manuscript received 16 January 2016; published 11 March 2016)
}

\begin{abstract}
We present theoretical and numerical results for the one-dimensional stochastically forced Burgers equation decimated on a fractal Fourier set of dimension $D$. We investigate the robustness of the energy transfer mechanism and of the small-scale statistical fluctuations by changing $D$. We find that a very small percentage of modereduction $(D \lesssim 1)$ is enough to destroy most of the characteristics of the original nondecimated equation. In particular, we observe a suppression of intermittent fluctuations for $D<1$ and a quasisingular transition from the fully intermittent $(D=1)$ to the nonintermittent case for $D \lesssim 1$. Our results indicate that the existence of strong localized structures (shocks) in the one-dimensional Burgers equation is the result of highly entangled correlations amongst all Fourier modes.
\end{abstract}

DOI: 10.1103/PhysRevE.93.033109

\section{INTRODUCTION}

An outstanding challenge of the past few decades has been to develop a rigorous understanding of the energy transfer from large to small scales in fully developed, threedimensional, incompressible turbulent flows [1]. Numerical simulations and experiments show that multipoint velocity correlation functions are intermittent, i.e., they develop a power-law behavior with nondimensional (anomalous) scaling exponents [1-3]. The question of the origins of intermittency in turbulence and its relation inter alia to small structures is also of central importance in the areas of nonequilibrium statistical physics, fluid dynamics, astrophysics, and geophysics [4-15].

In this paper, we investigate the small-scale properties of the stochastically forced one-dimensional Burgers equation, which is a paradigmatic example of a highly intermittent system with statistics dominated by coherent structures in physical space (shocks) $[16,17]$. For this, we perform a series of numerical experiments by studying the evolution of the original partial differential equation restricted on a fractal set of Fourier modes [18]. The idea is to reduce the number of degrees of freedom with minimal breaking of the original symmetries of the system. The goal is to understand what are the key ingredients in the dynamics necessary to reproduce the main statistical properties of the original nondecimated Burgers equation and therefore to understand the robustness and origins of its shock-like structures.

Over the past few decades various models of intermittency have been developed based on the idea of energy cascade in physical space [1]. These contrast with other attempts, based on the spectral space, involving statistical closures and renormalization group methods. Despite their success for certain problems, none of these attempts have been able to quantitatively connect anomalous scaling with the structure of the original equation, and hence with its intermittent

\footnotetext{
*buzzicotti.m@gmail.com

†biferale@roma2.infn.it

${ }^{\ddagger}$ uriel@oca.eu

§samriddhisankarray@gmail.com
}

behavior. As a result, our understanding of anomalous scaling is still based on phenomenological real-space descriptions and real-space methodologies.

Intermittency is intimately connected with ideas of energy transfer from large to small scales. Working in Fourier space should, hopefully, open new and possible ways to understand this cascade. In a recent work [18], the idea of fractal decimation was introduced, with the aim of studying the evolution of the Navier-Stokes equations on an effective dimension $D$ out of an integer $d$-dimensional embedding manifold. This is done by introducing a quenched mode-reduction in Fourier space such that in a sphere of radius $k$ the number of modes that are involved in the dynamics scale as $k^{D}$ (where $D<d$ is the effective fractal dimension of the system) for large $k[18,19]$. This approach allows us to decimate the number of triad interactions in Fourier space as a function of the wave numbers involved as well as to consider the problem in noninteger, fractal dimensions $D$. In Ref. [20] the first results for a set of simulations of the decimated, three-dimensional (3D) Navier-Stokes equation have been reported with the intriguing conclusion that fractal Fourier decimation leads, rather quickly (i.e., for a very small reduction of the Fourier modes $D \lesssim 3$ ), to vanishing intermittency.

In the present paper we investigate the same problem for the one-dimensional Burgers equation. The main advantage with respect to the previous attempt on the 3D Navier-Stokes is that here, due to the simpler structure of the problem, numerical simulations can reach much higher resolutions and therefore assess, in a fully quantitative way, the problem of scaling and corrections to it.

The rest of the paper is organized as follows. In Sec. II we introduce the decimated Burgers equation and give details about our numerical simulations. We then present results from our numerical simulations as well as provide theoretical and phenomenological arguments to substantiate them. In particular, in Sec. III we examine the effect of decimation on second-order correlation functions both in Fourier space, via the energy spectra (Sec. III A), and in physical space (Sec. III B) through the second-order structure function and flatness. We then, in Sec. IV, investigate in detail-by using numerical simulations and theory-the suppression of 
TABLE I. $D$, system dimension; $D=1$ denotes the ordinary nondecimated Burgers equation [Eq. (2)], while $D<1$ represents the decimated system as described in Eq. (3). $N$, number of collocation points. $\%(D)$, percentage of decimated wave numbers, where the first value is related to the lower resolution used while the second value is related to the higher one. $v$, value of the kinematic viscosity. $k_{f}$, the range of forced wave numbers. $C_{f}$, the mean energy injection, $\langle u f\rangle . N_{\text {mask }}$, number of different random quenched masks. $d t$, time step used in the temporal evolution.

\begin{tabular}{|c|c|c|c|c|c|c|c|}
\hline$D$ & $N$ & $\%(D)$ & $v$ & $k_{f}$ & $C_{f}$ & $N_{\text {mask }}$ & $d t$ \\
\hline 1 & $2^{16}-2^{18}$ & 0 & $8.0 \times 10^{-5}$ & {$[1: 5-10]$} & $0.01-0.05$ & 0 & $5.5 \times 10^{-5}$ \\
\hline 0.99 & $2^{16}-2^{19}$ & $8-10$ & $2.5 \times 10^{-5}$ & {$[1: 5-10]$} & $0.01-0.05$ & 32 & $2.3 \times 10^{-5}$ \\
\hline 0.97 & $2^{16}-2^{18}$ & $23-27$ & $9.0 \times 10^{-6}$ & {$[1: 5-10]$} & $0.01-0.05$ & 64 & $2.0 \times 10^{-5}$ \\
\hline 0.95 & $2^{16}-2^{18}$ & $36-40$ & $5.0 \times 10^{-6}$ & {$[1: 5-10]$} & $0.01-0.05$ & 64 & $1.7 \times 10^{-5}$ \\
\hline 0.90 & $2^{16}-2^{18}$ & $59-64$ & $2.0 \times 10^{-6}$ & {$[1: 5-10]$} & $0.01-0.05$ & 64 & $1.6 \times 10^{-5}$ \\
\hline 0.80 & $2^{16}-2^{18}$ & $83-87$ & $8.0 \times 10^{-7}$ & {$[1: 5-10]$} & $0.01-0.05$ & 96 & $1.5 \times 10^{-5}$ \\
\hline 0.70 & $2^{16}-2^{18}$ & 93-95 & $6.5 \times 10^{-7}$ & {$[1: 5-10]$} & $0.01-0.05$ & 96 & $1.5 \times 10^{-5}$ \\
\hline
\end{tabular}

intermittency by examining the higher-order structure functions. Finally, in Sec. V we make concluding remarks and provide a plausible theoretical framework in which to understand the spectral scaling seen in our simulations.

\section{THE BURGERS EQUATION ON A FRACTALLY DECIMATED FOURIER SET}

Following Ref. [18] we define the fractal Fourier decimation operator $P_{D}$ acting on a generic field $u(x, t)=\sum_{k} e^{i k x} \hat{u}_{k}(t)$ as

$$
v(x, t)=P_{D} u(x, t)=\sum_{k} e^{i k x} \theta_{k} \hat{u}_{k}(t),
$$

where $\theta_{k}$ are independently chosen random numbers, with $\theta_{k}=$ $\theta_{-k}$, such that $\theta_{k}=1$, with probability $h_{k}$ and $\theta_{k}=0$, with probability $1-h_{k}$. By choosing $h_{k}=k^{D-1}$, with $0<D \leqslant 1$, we introduce a quenched disorder that suppresses, randomly, modes on the Fourier lattice. On average we have $N(k) \propto k^{D}$ surviving modes at a distance $k$ from the origin. Considering $u$ as the velocity field given by the solution of the forced, one-dimensional Burgers equation,

$$
\frac{\partial u}{\partial t}+\frac{1}{2} \frac{\partial u^{2}}{\partial x}=v \frac{\partial^{2} u}{\partial x^{2}}+f,
$$

where $v$ is the viscosity, $u$ is $2 \pi$ space-periodic in $x$, and $f$ is a stochastic force acting on a few shells, which drives the system to a statistical steady state. We can then write the decimated Burgers equation, which gives the evolution for the decimated field $v$ as

$$
\frac{\partial v}{\partial t}+\frac{1}{2} P_{D} \frac{\partial v^{2}}{\partial x}=v \frac{\partial^{2} v}{\partial x^{2}}+f,
$$

with initial conditions $v_{0}=P_{D} u_{0}$ [21]. When $D=1$ we recover the usual one-dimensional equation. It is important to notice that the fractal projector in front of the nonlinear term in Eq. (3) is fundamental to ensure that the nonlinear convolution does not activate the decimated degrees of freedom during the system dynamics. It is interesting to note that the fractal decimation, as well as any other Galerkin truncation, preserves the inviscid conservation of the first three moments of the velocity field only.

We performed a set of numerical simulations of Eq. (3) by changing the dimension $D$ between $0.70 \leqslant D \leqslant 1.0$ and the number of collocation points $N$ from $2^{16}$ to $2^{19}$. We choose the forcing to be Gaussian and white-in-time, such that $\left\langle\hat{f}\left(k_{1}, t_{1}\right) \hat{f}\left(k_{2}, t_{2}\right)\right\rangle=f_{0}\left|k_{f}\right|^{-1} \delta\left(t_{1}-t_{2}\right) \delta\left(k_{1}+k_{2}\right)$, acting only on a shell of wave numbers at large scales $k_{f} \in[1$ : 5-10]. We use a pseudospectral method with a second-order Adams-Bashforth scheme to integrate in time. Details of all simulations can be found in Table I. We note that the values of $v$ decreases with the dimension $D$ (see Table I); indeed as the decimation becomes stronger the contribution of the nonlinear advection term becomes weaker [18]. Hence, to compare results from simulations with different values of $D$, we use smaller and smaller values of $v$ in order to observe a similar extension of the inertial range. We do not know if the decimated equations are well behaved as $v \rightarrow 0$ and if the system develops a dissipative anomaly leading to a stationary behavior for all $D$. This is an interesting point that will be addressed in future work.

Hereafter we analyze statistical properties for either a single quenched realization of the decimation mask or after a further averaging over different realizations of the quenched disorder. We indicate with $\langle\bullet\rangle$ the average over time for a single realization of the decimation mask; while $\boldsymbol{-}$ is used to denote an average over different quenched masks, where each mask acts as a projector.

It is well known that the ordinary forced Burgers equation develops several discontinuities (shocks) connected by smooth, continuous ramps as it evolves in time [see Fig 1(a)]. As soon as we introduce the fractal decimation projector, several sharp oscillatory structures appear in the solution for $v(x, t)$, even for mild decimation $(D \lesssim 1)$, as seen in Fig. 1(b). Such structures, although reminiscent of features (tygers) of the Galerkin-truncated Burgers equation [22], are crucially different because they are spatially much more delocalized.

\section{SECOND-ORDER FUNCTIONS}

\section{A. Scaling in Fourier space: The energy spectra}

The first question we want to address is the effect of decimation on the mean spectral properties. We define the energy spectrum for a general fractal dimension as $E_{k}=\theta_{k}\left\langle\hat{u}_{k}^{2}\right\rangle$. Since fractal decimation does not break scaling invariance of the original equation in the inviscid limit, we still expect to 

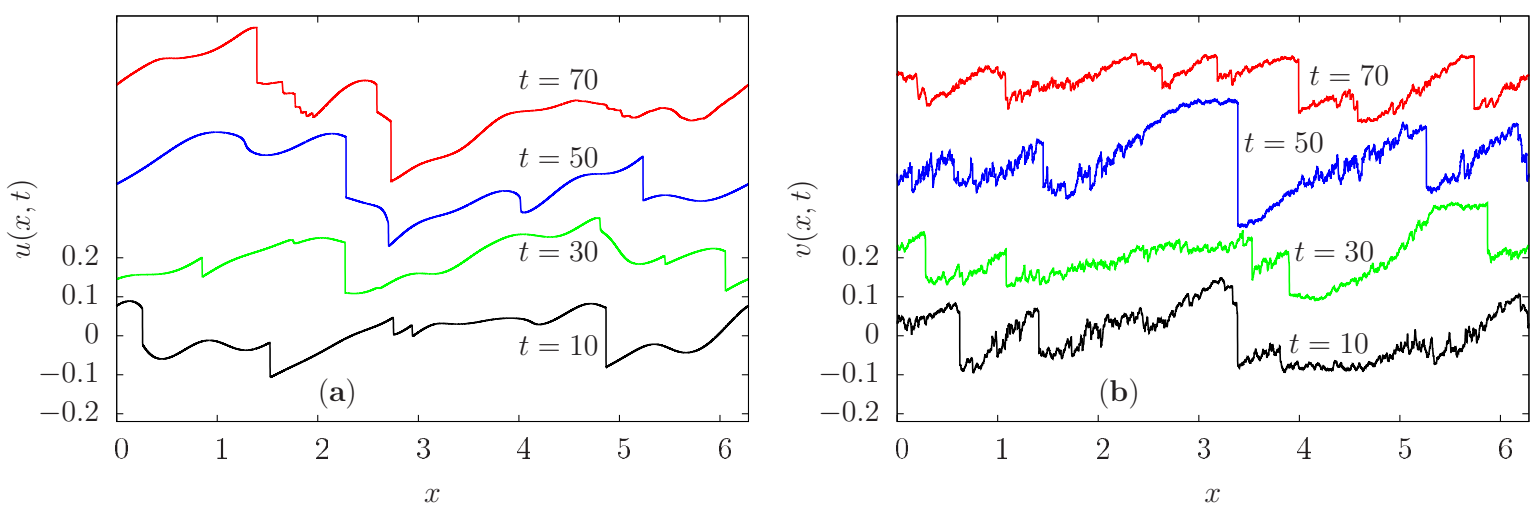

FIG. 1. Representative plots of the solutions of the stochastically forced (a) Burgers equation and (b) the decimated Burgers equation for fractal dimension $D=0.99$ at times $t=10, t=30, t=50$, and $t=70$ (respectively from lower black (black) line to upper red (gray) line). The velocities at different time are shifted upward on the $y$ axes.

observe a power-law dependency as a function of the wave number $E_{k} \sim k^{\alpha_{D}}$. Another important quantity is the spectrum averaged over the quenched disorder:

$$
\bar{E}_{k}=\overline{\left\langle\hat{u}_{k}^{2}\right\rangle}=\frac{1}{N_{\text {mask }}} \sum_{n=1}^{N_{\text {mask }}} \theta_{k}^{(n)}\left\langle\hat{u}_{k}^{2}\right\rangle,
$$

where with $n=1, \ldots, N_{\text {mask }}$ we indicate different realizations of the decimation mask. From these definitions, one can infer the following relations:

$$
\bar{E}_{k} \simeq k^{D-1} E_{k} \sim k^{\beta_{D}} ; \quad \beta_{D}=\alpha_{D}+D-1,
$$

where we have assumed that the scaling properties of the velocity field depend only on the fractal dimension $D$ but are independent of the particular $n$th realization of the decimation mask. To ensure the validity of Eq. (4) we need to use $N_{\text {mask }}$ large enough to smooth out the gaps produced by the different masks in the energy spectra.

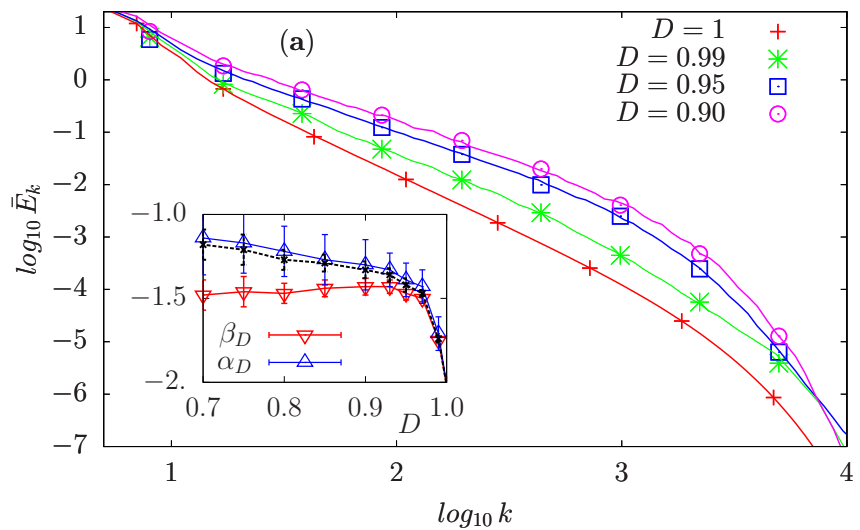

For the one-dimensional Burgers equation, because of the presence of the $\operatorname{shock}(\mathrm{s})$, the energy spectrum scales as $\bar{E}_{k}=$ $E_{k} \sim k^{-2}$.

In Fig. 2(a) a log-log plot of $\bar{E}_{k}$ versus $k$ for $0.9 \leqslant D \leqslant$ 1.0 is shown. The mean energy spectrum $\bar{E}_{k} \sim k^{\beta_{D}}$ becomes shallower when decreasing $D$. In the inset of Fig. 2(a), we show the dependency of the scaling exponents on $D$. It changes from $\beta_{D}=-2$ for $D=1$ to $\beta_{D}=-3 / 2$ for $D \lesssim 0.97$, with a sharp transition around $D \sim 0.97$. In the same inset we also show the validity of the scaling relation Eq. (4).

The existence of this quasisingular behavior for the spectral slope at $D \sim 1$ might indicate the presence of an intermediate asymptotics spoiling the true behavior in the limit of vanishing viscosity. To confirm this possibility, we show, in Fig, 2(b), the energy spectrum $\bar{E}_{k}$ versus $k$ for $D=0.99$ at various values of the viscosity and resolution. It is seen that there is a trend, when decreasing viscosity, toward the development of an inertial

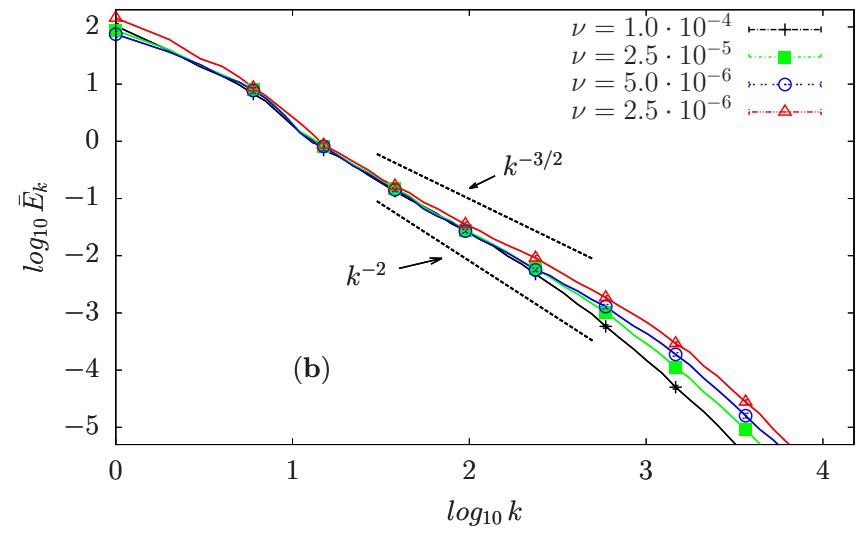

FIG. 2. (a) Mean energy spectrum, $\bar{E}_{k}$ as a function of the wave numbers $k$; different lines and symbols represent different fractal dimensions $D$ (see the legend for details). Inset: the scaling exponents $\beta_{D}$ (red line, downward triangles) for the mean energy spectrum as a function of the dimension $D$; exponents $\alpha_{D}$ (blue line, upward triangles) obtained from the spectrum for a single projector. The latter is computed by averaging over the exponents obtained from each individual projector. The black dashed line confirms the relation $\beta_{D}+1-D=\alpha_{D}$ as obtained in Eq. (4). The error bars for $\beta_{D}$ are obtained by halving the set of projectors used in the computation of the mean energy spectrum, while the error bars for $\alpha_{D}$ are the standard deviation among all the values used in the calculation of the mean exponent. (b) Mean energy spectrum (error bars are inside the symbols) at $D=0.99$ for the following resolutions and values of the viscosity (see legend as well): $v=1.0 \times 10^{-4}, N=2^{17}$ (black line); $v=2.5 \times 10^{-5}, N=2^{18}$ (green squares and line); $v=5.0 \times 10^{-6}, N=2^{18}$ (blue circles with line); and $v=2.5 \times 10^{-6}, N=2^{19}$ (red triangles with line). The dashed black lines represent the scaling $k^{-3 / 2}$ and $k^{-2}$ as a guide to the eye. 

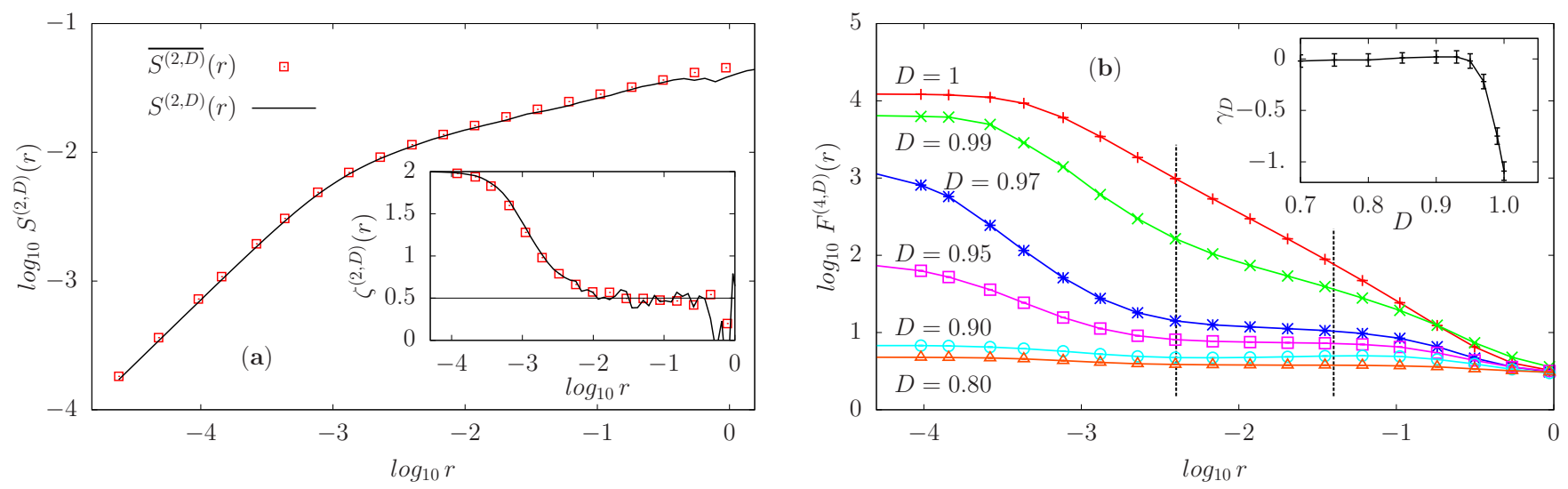

FIG. 3. (a) $\overline{S^{(2, D)}}(r)$ (red squares) and $S^{(2, D)}(r)$ (black solid line) measured at $D=0.80$ and (inset) their associated local slopes [Eq. (8)]. (b) The flatness, $F^{(4, D)}(r)$ versus $r$ (log-log scale) for different dimensions $D$. The fit is done in the range: $0.004 \leqslant r \leqslant 0.04$ as illustrated by the two vertical dotted lines to yield the exponent $\gamma_{D}$ as shown in the inset.

range scaling $\bar{E}_{k} \sim k^{-3 / 2}$. This suggests that, asymptotically, as $v \rightarrow 0$, the true scaling exponent $\beta_{D} \rightarrow-3 / 2$ for any $D<1$. This observation would imply that the continuous transition seen in the insets of Fig. 2(a) is a consequence of some intermediate asymptotics and that Fourier decimation is a singular perturbation for the spectral scaling properties.

\section{B. Scaling in physical space}

In order to substantiate the relation between the change in the spectrum and the suppression of shocks we analyze the physical space velocity field. This is also required to address the issue of anomalous scaling due to the lack of a clear definition of intermittency in Fourier space.

Intermittent features in turbulent flows are quantified by the statistics of multipoints correlation function or the so-called structure functions:

$$
S^{(p, D)}(r)=\left\langle\left\langle\delta_{r} v^{p}\right\rangle\right\rangle_{x, t},
$$

where $\delta_{r} v=v(x+r)-v(x)$ and the angular brackets, $\langle\langle\bullet\rangle\rangle_{x, t}$, denote space and time averaging over the statistically stationary state. It is important to remark that the spatial average is equivalent to an average over the quenched disorder. To prove this we notice that

$$
\begin{aligned}
\overline{S^{(2, D)}}(r) & =\int d x(\bar{v}(x+r)-\bar{v}(x))^{2}=\int d k\left(e^{i k r}-1\right) \bar{E}_{k} \\
& =\frac{1}{N_{\text {mask }}} \sum_{n=1}^{N_{\text {mask }}} \int d k\left(e^{i k r}-1\right) \theta_{k}^{(n)} E_{k}=S^{(2, D)}(r),
\end{aligned}
$$

where we have used the scaling properties of the probability defining the decimation mask and the fact that $d k \theta_{k}^{(n)} \stackrel{\text { law }}{\sim}$ $d k k^{D-1}$, where the symbol $\stackrel{\text { law }}{\sim}$ stands for statistically "in law"; i.e., the two sides have the same scaling properties when averaged on different realizations of the fractal projector. This relation is validated in Fig. 3(a), where the secondorder structure functions obtained both from a single mask (continuous black line) and from an average over different realizations of the quenched masks (square symbols in red) are shown to be identical. For this reason henceforth, we stop distinguishing between $\bar{S}_{p}(r)$ and $S_{p}(r)$. To understand the effects of decimation on intermittency, we measure the flatness of structure functions:

$$
F^{(4, D)}(r)=\frac{S^{(4, D)}(r)}{\left[S^{(2, D)}(r)\right]^{2}} \sim r^{\gamma_{D}},
$$

as a function of $r$ for different values of $D$. Let us stress that for the 1D Burgers equations, phenomenological and theoretical arguments [17] predict $\zeta(p)=\min (p, 1)$ (see Fig. 2), which give for the flatness the scaling $r^{-1}$ [Fig. 3(b), inset]. As shown in Fig. 3(b) we find that scaling exponents, $\gamma_{D}$, present the same sharp transition already observed in the slope of the energy spectra for $1 \leqslant D \leqslant 0.97$ [Fig. 2(a), inset]. Thus, surgeries on the Fourier space and dimensional reduction seem to suppress intermittency in hydrodynamics (as has also been seen in Ref. [20]). We cannot refrain from noticing that this seems to be in contrast with the usual phenomenology of cascade dynamics, built in terms of local-Fourier interactions.

\section{HIGHER-ORDER STATISTICS}

The results obtained in the previous section lead us to address the question of whether intermittency is indeed washed out by any small perturbation of the Fourier dynamics-bad news for modeling-or if it is masked by new leading fluctuations introduced by the modified nonlinear dynamics. To answer this question, we perform a systematic analysis of the scaling properties of structure functions by changing the fractal Fourier dimension $D$. It is important to decompose the structure function into contributions from the negative and positive increments of the velocity field [17]. We thus define

$$
S_{+}^{(p, D)}(r)=\left\langle\left\langle\left(\delta_{r}^{+} v\right)^{p}\right\rangle\right\rangle_{x, t} ; \quad S_{-}^{(p, D)}(r)=\left\langle\left\langle\left(\delta_{r}^{-} v\right)^{p}\right\rangle\right\rangle_{x, t},
$$

where $\delta_{r}^{+} v \equiv \delta_{r} v>0$ and $\delta_{r}^{-} v \equiv \delta_{r} v<0$, whence the structure function $S^{(p, D)}(r)=S_{+}^{(p, D)}(r)+(-1)^{p} S_{-}^{(p, D)}(r)$. To 

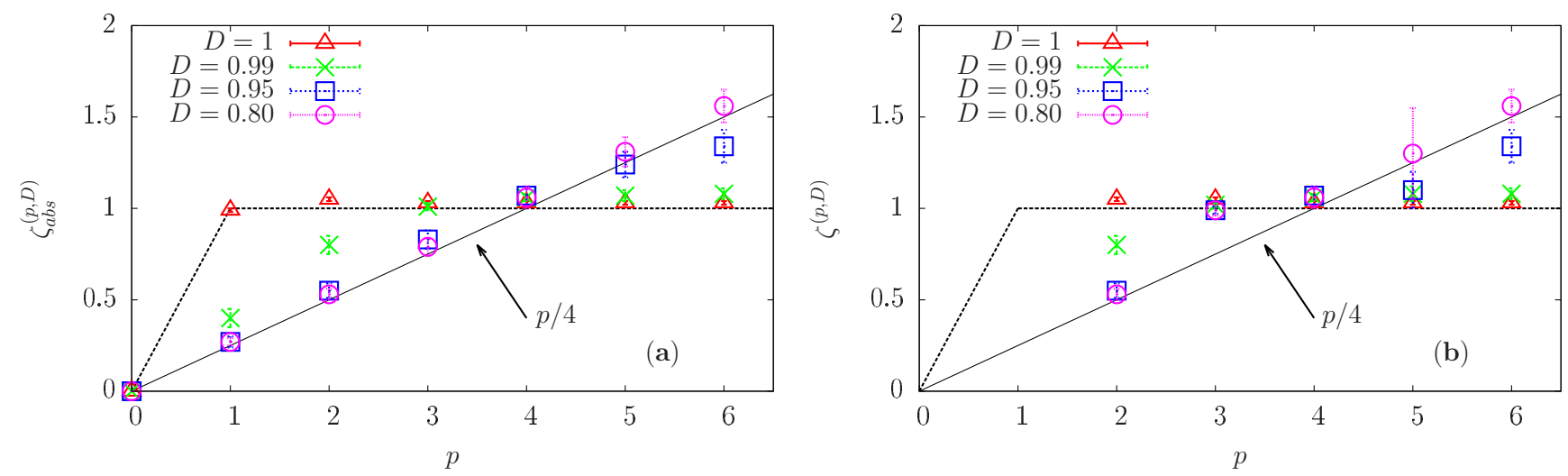

FIG. 4. Inertial range scaling exponents (a) $\zeta_{\mathrm{abs}}^{(p, D)}$ and (b) $\zeta^{(p, D)}$ for the structure functions (a) with and (b) without the use of absolute values versus the order $p$; the different symbols are related to different dimensions $D$ (see legend), the dashed lines are the bifractal behavior of the $1 D$ Burgers equation. The values of the exponents are estimated as the best fit of local scaling exponents and the error bars are estimated from the variations of the local scaling exponents within the fitting range. We note that $\zeta_{\mathrm{abs}}^{(3, D)}$ does not satisfy the $1 D$ Kármán-Howarth analytical relation because of the competition between the leading and subleading terms introduced by the decimation in the scaling of the velocity field; in contrast, the Karman-Howarth analytical relation is satisfied for the case without the absolute values (see text).

improve the statistics, odd-order structure functions are often measured in terms of the absolute value of velocity increments; in this case we will obviously have $S_{\mathrm{abs}}^{(p, D)}(r)=S_{+}^{(p, D)}(r)+$ $S_{-}^{(p, D)}(r)$. To study the scaling properties it is customary to analyze logarithmic local slopes:

$$
\begin{aligned}
& \zeta_{\mathrm{abs}}^{(p, D)}(r)=\frac{d \log S_{\mathrm{abs}}^{(p, D)}(r)}{d \log r}, \\
& \zeta^{(p, D)}(r)=\frac{d \log S^{(p, D)}(r)}{d \log r} .
\end{aligned}
$$

The scaling exponents of order $p$ in the inertial range are obtained as a best fit to the local exponents in the interval of scales where they are close to a constant. In Fig. 4 we show the result for both $\zeta_{\mathrm{abs}}^{(p, D)}$ and $\zeta^{(p, D)}$ (see figure captions for details).

From a comparison of the two figures one can conclude a few important facts. First, there is a clear tendency for even-order moments to approach the nonintermittent scaling behavior with exponent $p / 4$ as soon as $D<1$; the agreement being almost perfect already at $D \leqslant 0.95$. Second, the oddorder moments of the structure functions, defined without absolute values, seem to maintain a memory of the original nondecimated Burgers behavior, namely $\zeta(p, D)=1 \forall p \geqslant 1$, even for small fractal dimensions $D \ll 1$. Let us note that this behavior is not entirely unexpected. First, the emergence of a linear scaling $p / 4$ is in agreement with the observation of the spectral slope $\beta_{D}=-3 / 2$ and with the absence of intermittency. Figure 4 (a) is thus a demonstration of the suppression of intermittency in Burgers flows under fractal Fourier decimation, similar to what has been observed for the Navier-Stokes evolution in Ref. [20]. The deviation from the values $p / 4$ for the odd-order moments is explained by noticing that the third-order structure function must satisfy an analytical relation similar to the Kármán-Howarth 4/5 law of Navier-Stokes, namely, $S^{(3, D)}(r)=-6 \epsilon r$ for all $D$ and where $\epsilon$ is the mean energy dissipation. Indeed, Fig. 4(b) clearly supports this statement. A possible way to rationalize these apparently contradictory results is to suppose that decimation introduces a distributed noise at all scales, leading
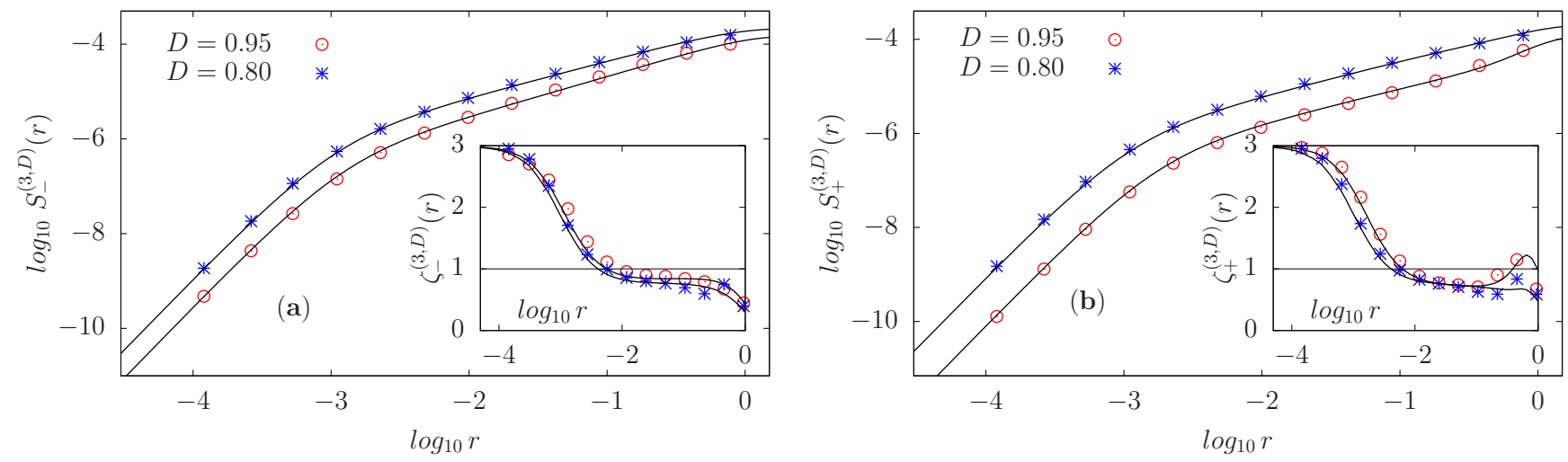

FIG. 5. (a) Structure functions for $D=0.80$ (blue stars) and $D=0.95$ (red circles) for (a) the negative increments $S_{-}^{(3, D)}(r)$ and (b) positive increments structure functions $S_{+}^{(3, D)}(r)$; the black solid lines are the respective fitting functions [Eq. (10)]. In the insets, with the same legend, we show the associated local slopes. 

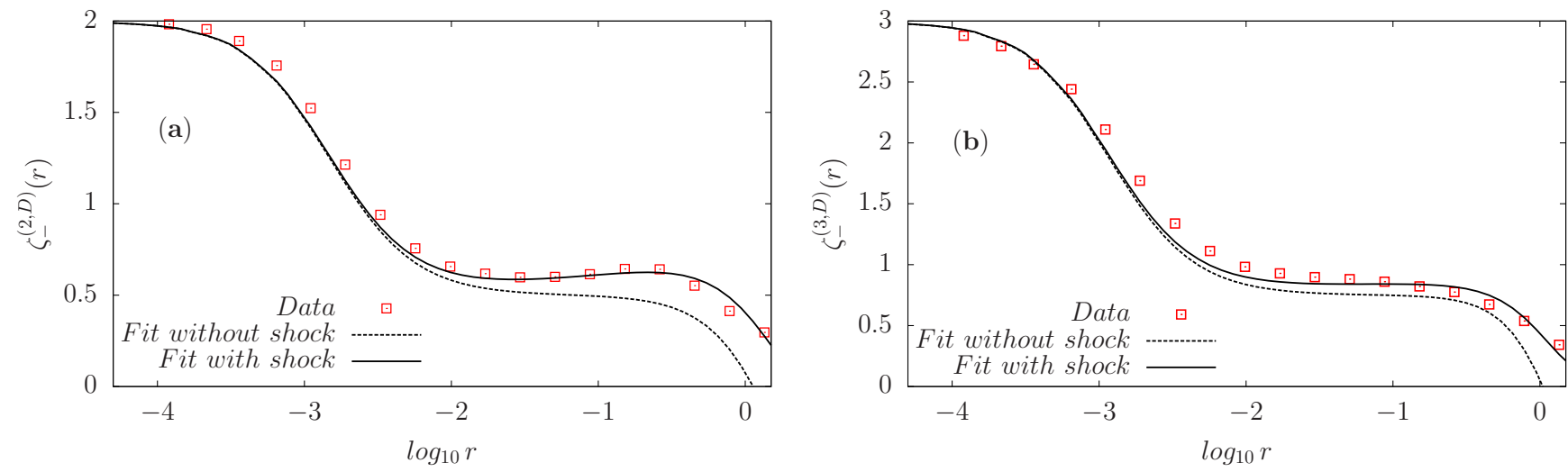

FIG. 6. (a) Local slopes of the structure functions associated with the negative increments at $D=0.95$ for $\left(\right.$ a) the second order $\left[S_{-}^{(2, D)}(r)\right]$ and (b) the third order $\left[S_{-}^{(3, D)}(r)\right]$; the black solid lines are obtained from the fitting function Eq. (10) and the dashed lines from the fit obtained without the shock contribution by setting $B_{+,-}^{(p, D)}=0$ in Eq. (10).

to a typical scaling $\delta_{r} v \propto r^{1 / 4}$ on top of an underlying Burgers-like dynamics. If this is true, it should be detectable by looking separately at positive or negative velocity increments. As a result, we suggest the presence of two different asymptotics:

$$
\begin{aligned}
& S_{+}^{(p, D)}(r)=r^{p / 4}+\text { smooth, } \\
& S_{-}^{(p, D)}(r)=r^{p / 4}+r+\text { smooth, }
\end{aligned}
$$

where the first term on the right-hand side of the equations should have prefactors that go to zero for $D \rightarrow 1$. In Eq. (9) the Burgers scaling $\propto r$ is present only for the negative increments and smooth denotes the subleading differentiable terms induced by the viscous contribution $\propto r^{p}$.

In order to quantitatively check the above prediction, we perform a series of systematic fits to $S_{ \pm}^{(p, D)}(r)$ by using the following interpolation expression for the asymptotic

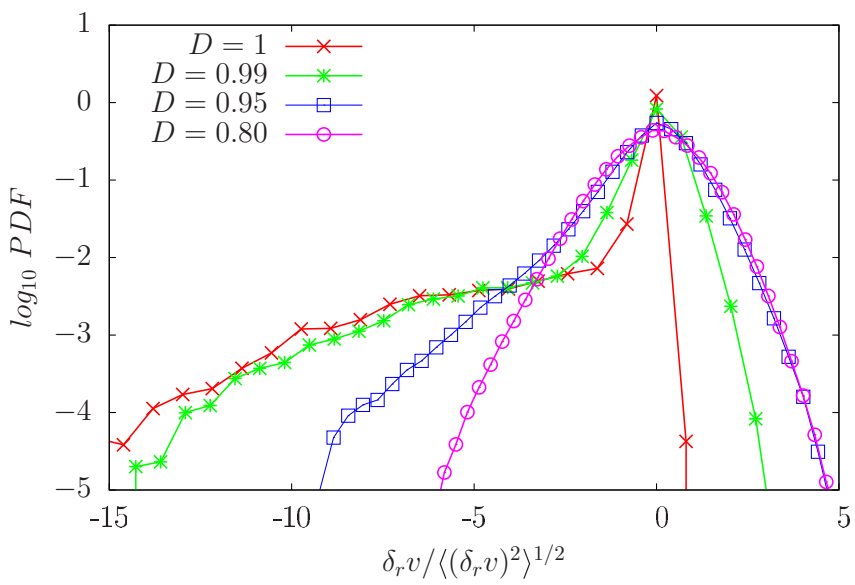

FIG. 7. The probability density function (PDF) of the velocity increments at a scale $r \sim 0.005$; the PDF is normalized by its standard deviation. The different lines correspond to different dimensions $D$ as shown in the legend. behavior (9):

$$
\begin{aligned}
S_{+}^{(p, D)}(r)= & \left(\frac{A_{+}^{(p, D)} r^{p}}{\left(r^{2}+\eta^{2}\right)^{(p-p / 4) / 2}}+B_{+}^{(p, D)} r^{p}\right)\left(1+\frac{r}{L}\right)^{c_{+}}, \\
S_{-}^{(p, D)}(r)= & \left(\frac{A_{-}^{(p, D)} r^{p}}{\left(r^{2}+\eta^{2}\right)^{(p-p / 4) / 2}}+\frac{B_{-}^{(p, D)} r^{p}}{\left(r^{2}+\eta^{2}\right)^{(p-1) / 2}}\right) \\
& \times\left(1+\frac{r}{L}\right)^{c_{-}},
\end{aligned}
$$

where $A_{ \pm}^{(p, D)}, B_{ \pm}^{(p, D)}$ are fitting constants and $\eta$ is the dissipative scale such that for $r<\eta, S^{(p, D)}(r) \propto r^{p}$. The overall factor $(1+r / L)^{c_{ \pm}}$is used to saturate the inertial range scaling for $r$ beyond the forcing length scale. $L$ and $c_{ \pm}$are estimated as the best-fitting parameters. From Eq. (10) it is easy to recognize that the first term on the right-hand side of both equations represents the effect introduced by the decimation, while the second term (present only for negative increments) represents the standard shock-dominated Burgers scaling (plus viscous contributions). Clearly, if $A_{+} \sim A_{-}$for all $D$, we have moments of even order that are dominated by the $p / 4$ scaling while moments of odd order have the usual Burgers scaling. In Figs. 5(a) and 5(b) we show the best fit by using these expressions for the third-order moment $(p=3)$ and $D=0.95,0.8$ for both the (a) negative and (b) positive increments. For the case of negative increments, we also show the best fit with and without the shock contribution to highlight the importance of the shock to reconstruct the right behavior for $p=2$ in Fig. 6(a) and for $p=3$ in Fig. 6(b). As one can see, the Eqs. (10) are able to reconstruct the local scaling properties in a robust way, showing that our phenomenological model is not incompatible with the data. Finally, we measure the probability density function of the velocity increments at different scales (Fig. 7). This clearly shows the emergence of nontrivial fluctuations for positive velocity increments at decreasing fractal dimension $D$; such fluctuations are almost absent in the standard one-dimensional Burgers case. In summary, all our results indicate that while on the one hand the numerical evidence point toward a robustness of the shock structure (as also visually confirmed from Fig. 1), on the 
other hand, decimation introduces important fluctuations that spoil the scaling of the original undecimated equation without modifying the existence of a constant flux of energy from large to small scales.

\section{CONCLUSIONS}

Let us now turn to a few theoretical considerations for understanding the behavior of $\zeta^{(p, D)}$. We first recall that just like any Galerkin truncation [22], the fractal Fourier decimation constrains the number of conserved quantities to the first three moments of the velocity in the Burgers equation. In particular, this allows the conservation of a cubic moment whose relative flux would yield $\zeta_{4}=1$, which, in turn, would be consistent with our numerical result $\zeta_{p}=p / 4$. Another possible explanation for the $E(k) \propto k^{-3 / 2}$ scaling is the idea that a new decorrelation mechanism in the shell-to-shell energy transfer across Fourier modes might be introduced by the fractal decimation. The fractal mask can be seen as an extra, ad-hoc removal of nonlinear couplings at all scales and, as such, a sort of power-law external "energy-conserving" noise. It is not unphysical to suppose that due to the power-law dependence on the wave number, a different weight between local and nonlocal interactions in Fourier space is introduced, making the latter more important than in the usual $D=1$ case. Given all this, it is conceivable that an extra decorrelation time of the order of $\tau_{\operatorname{dec}}(k) \propto 1 / k$ appears, leading to a slow down of the energy transfer mechanisms, as is the case for Alfvén waves in MHD [23] or in the presence of a rapid distortion mechanism [24]. Typically, this leads to an estimate for the energy flux $\epsilon=k E(k) / \tau_{\text {tr }}(k)$, where the transfer time is given in terms of a golden mean between the eddy-turn-over time $\tau_{\text {eddy }}(k)$ and the decorrelation time $\tau_{\text {dec }}(k)$, $\tau_{\text {tr }}(k)=\tau_{\text {eddy }}^{2} / \tau_{\text {dec }}(k)$. If this is the case, considering that $\tau_{\text {eddy }} \propto\left[k^{3} E(k)\right]^{-1 / 2}$, we arrive at the estimate $E(k) \propto k^{-3 / 2}$.

Let us note that other decimation protocols might be imagined. In particular, one can consider performing a selective decimation of a single class of triads (e.g., local or nonlocal), in order to probe the main mechanisms leading to the formation of small-scale shocks in the dynamical evolution. In this case, decimation cannot be univocally defined in terms of each wave number; i.e., one wave number might belong to a local or nonlocal triads depending on the other two. Hence, a selected triads reduction can be done only inside the nonlinear convolution term, accessible via a fully spectral code with strong limitation in the numerical resolution achievable; see Ref. [25].

Moreover, a recent study [26] has shown that highly nontrivial time correlations among Fourier triads are connected to the presence of intermittency in physical space. It is not obvious a priori that reducing Fourier interaction will lead to a time desynchronization of the energy exchange among triads. The results of Ref. [26] together with the ones shown here suggest that the build up of small-scale intermittent fluctuations in physical space (shocks) is indeed the outcome of an entangled temporal correlations amongst many (all?) Fourier modes. Another interesting potentially useful methodology is to apply proper orthogonal decomposition of Fourier amplitudes and phases correlations [27].

In this paper, we have presented a set of theoretical and numerical results concerning the evolution of the onedimensional stochastically forced Burgers equation decimated on a fractal Fourier set. Decimation leads, very quickly, to a suppression of the shock-dominated statistics, indicating that the bifractal scaling properties of the original equation are very sensitive to the details of the dynamical evolution. Similar results have also been recently obtained for the more complicated case of the dynamics of fully developed incompressible turbulent flows in three-dimensional Navier-Stokes equations. Some properties connected to the existence of shock-like solutions are nevertheless robust, but subleading. Our results indicate that the existence of strong localized fluctuations in Burgers is the result of highly entangled correlations among all Fourier modes. This might be important to develop models for the nonlinear evolution based on suitable reduction (and replacement) of a subset of the original degrees of freedom.

\section{ACKNOWLEDGMENTS}

M.B. and L.B. acknowledge funding from the European Research Council under the European Union's Seventh Framework Programme, ERC Grant Agreement No. 339032. L.B. and S.S.R. thank Cost Action MP1305 for support. S.S.R. acknowledges the support of the Indo-French Center for Applied Mathematics (IFCAM) and Airbus Group Corporate Foundation Chair in Mathematics of Complex Systems established in ICTS and the hospitality of the Department of Physics, University of Rome "Tor Vergata," Rome, Italy, and the Observatoire de la Côte d'Azur, Nice, France.
[1] U. Frisch, Turbulence: The Legacy of A. N. Kolmogorov (Cambridge University, Cambridge, UK, 1996).

[2] A. N. Kolmogorov, Dokl. Akad. Nauk SSSR 30, 301 (1941).

[3] G. Falkovich, K. Gawedzki, and M. Vergassola, Rev. Mod. Phys. 73, 913 (2001).

[4] J.-D. Fournier and U. Frisch, Phys. Rev. A 28, 1000 (1983).

[5] Intermittency in Turbulent Flows, ed. J. C. Vassilicos (Cambridge University, Cambridge, UK, 2001).

[6] J. Cho, A. Lazarian, and E. T. Vishniac, Astrophys. J. 564, 291 (2002).

[7] W.-C. Muller and D. Biskamp, Phys. Rev. Lett. 84, 475 (2000).
[8] P. Veltri, Plasma Phys. Controlled Fusion 41, A787 (1999).

[9] R. Grauer, J. Krug, and C. Marliani, Phys. Lett. A 195, 335 (1994).

[10] J. J. Riley and E. Lindborg, J. Atmos. Sci. 65, 2416 (2008).

[11] G. Boffetta and R. E. Ecke, Annu. Rev. Fluid Mech. 44, 427 (2012).

[12] R. Benzi, G. Paladin, G. Parisi, and A. Vulpiani, J. Phys. A: Math. Gen. 18, 2157 (1985).

[13] A. C. Newell, S. Nazarenko, and L. Biven, Physica D: Nonlin. Phenom. 152-153, 520 (2001).

[14] A. Joel Chorin, Commun. Pure Appl. Math. 34, 853 (1981) 
[15] U. Frisch and G. Parisi, Turbulence and Predictability in Geophysical Fluid Dynamics and Climate Dynamics, edited by M. Ghil, R. Benzi, and G. Parisi (North-Holland, Amsterdam, 1985), Vol. 88, pp. 71-88.

[16] U. Frisch and J. Bec, Les Houches 2000: New Trends in Turbulence, edited by M. Lesieur, A. Yaglom, and F. David (Springer EDP-Sciences, Berlin, 2001), pp. 341-383.

[17] J. Bec and K. Khanin, Phys. Rep. 447, 1 (2007).

[18] U. Frisch, A. Pomyalov, I. Procaccia, and S. S. Ray, Phys. Rev. Lett. 108, 074501 (2012).

[19] S. S. Ray, Pramana J. Phys. 84, 395 (2015).

[20] A. S. Lanotte, R. Benzi, S. K. Malapaka, F. Toschi, and L. Biferale, Phys. Rev. Lett. 115, 264502 (2015)
[21] The forcing is also projected on the decimated lattice.

[22] S. S. Ray, U. Frisch, S. Nazarenko, and T. Matsumoto, Phys. Rev. E 84, 016301 (2011).

[23] P. S. Iroshnikov, Sov. Astron. 7, 566 (1964); R. H. Kraichnan, Phys. Fluids 8, 1385 (1965).

[24] J. C. R. Hunt and D. J. Carruthers, J. Fluid Mech. 212, 497 (1990).

[25] M. Leslie Smith and Y. Lee, J. Fluid Mech. 535, 111 (2005).

[26] M. Buzzicotti, B. P. Murray, L. Biferale, and M. D. Bustamante, arXiv:1509.04450.

[27] P. Holmes, J. L. Lumley, and G. Berkooz, Turbulence, Coherent Structures, Dynamical Systems and Symmetry (Cambridge University Press, Cambridge, 1998). 